\title{
ASO Author Reflections: Radiopathomics Strategy of Combing Multi-scale Tumor Information on Pretreatment to Predict the Pathologic Response to Neoadjuvant Therapy
}

\author{
Jie Tian, $\mathrm{PhD}^{1,2,3,4} \mathbb{1}_{\mathbb{0}}$, Xinjuan Fan, $\mathrm{MD}^{5}$, Ruihua $\mathrm{Xu}, \mathrm{MD}^{6}$, Ying-Shi Sun, $\mathrm{MD}^{7}$, and Guanyu Yang, $\mathrm{PhD}^{8}$ \\ ${ }^{1}$ CAS Key Laboratory of Molecular Imaging, Institute of Automation, Beijing, China; ${ }^{2}$ School of Artificial Intelligence, \\ University of Chinese Academy of Sciences, Beijing, China; ${ }^{3}$ Beijing Advanced Innovation Center for Big Data-Based \\ Precision Medicine, School of Medicine, Beihang University, Beijing, China; ${ }^{4}$ Engineering Research Center of Molecular \\ and Neuro Imaging of Ministry of Education, School of Life Science and Technology, Xidian University, Xi'an, China; \\ ${ }^{5}$ Department of Pathology, the Sixth Affiliated Hospital, Sun Yat-sen University, Guangzhou, China; ${ }^{6}$ State Key \\ Laboratory of Oncology in South China, Collaborative Innovation Center for Cancer Medicine, Sun Yat-sen University \\ Cancer Center, Guangzhou, China; ${ }^{7}$ Key Laboratory of Carcinogenesis and Translational Research (Ministry of Education/ \\ Beijing), Department of Radiology, Peking University Cancer Hospital and Institute, Beijing, China; ${ }^{8}$ School of Computer \\ Science and Engineering, Southeast University, Nanjing, China
}

\section{PAST}

The standard treatment for locally advanced rectal cancer (LARC) includes neoadjuvant chemoradiotherapy (nCRT) followed by total mesorectal excision (TME) and adjuvant chemotherapy. ${ }^{1}$ After nCRT, $15-27 \%$ of patients with LARC achieve a pathologic complete response (pCR) and usually have perfect long-term outcomes. These patients prefer to avoid surgery and preserve organs with a strategy such as "watch and wait" management. ${ }^{2}$ Additionally, for more than $50 \%$ of patients who cannot reach a good response (GR), ${ }^{3}$ treatment optimization according to different pathologic responses is essential to balance the benefits of nCRT against toxicity. ${ }^{4}$ Due to the advantages of radiomics for quantitative analysis of tumors, ${ }^{5}$ radiomics has demonstrated the potential of magnetic resonance imaging (MRI) in preoperative accurate evaluation of $\mathrm{pCR}^{6}$ or no response ${ }^{7}$ in previous studies. Furthermore, pretreatment multi-parameter magnetic resonance imaging (mp-MRI)-based radiomics was attempted to predict non-

(C) The Author(s) 2020

First Received: 20 May 2020;

Published Online: 3 June 2020

J. Tian, $\mathrm{PhD}$

e-mail: jie.tian@ia.ac.cn response to nCRT. ${ }^{8}$ However, to date, no nomogram has been established or acknowledged for predicting discrepancies in the response before nCRT.

\section{PRESENT}

In this study, ${ }^{9} 981$ consecutive patients with evaluation of response according to tumor regression grade (TRG) who received nCRT (primary cohort and external validation cohorts 1-3) were retrospectively recruited from four Chinese hospitals. Each recruited patient had received both a pretreatment multi-parametric magnetic resonance imaging (mp-MRI) and a whole-slide image (WSI) of biopsy specimens. Quantitative image features were extracted from the mp-MRI and WSI. These features then were used for radiopathomics signature (RPS) construction powered by an artificial intelligence model. The predicted signature from the radiopathomics model yielded an overall accuracy (ACC) of 79.66-87.66\% in the validation cohorts (VCs). The areas under the curve (AUCs) of RPS at specific response grades were 0.98 (TRG0), 0.93 ( $\leq$ TRG1), and 0.84 ( $\leq$ TRG2). The RPS at each grade of pathologic response showed significant improvement over signature construction without combining multi-scale tumor information $(P<0.01)$. The authors' proposed radiopathomics strategy and signature escaped the limitation of using only medical imaging to depict the whole 
tumor and decreased the potential risk of overlooking tumor heterogeneity by adding micro-scale pathologic information.

\section{FUTURE}

This study provided evidence that the radiopathomics strategy of combing the images of both radiology and pathology is a potential strategy for predicting the variation in pathologic response before nCRT. More quantitative and multi-scale tumor information powered by the information fusion method was able to improve the heterogeneous description of tumors and enhance the performance of the model for restaging patients. As such, future studies should focus on the integration of gene and protein information to construct a more comprehensive tumor prediction model from the macro radiological information of tumor to the micro pathological information of tumor. The biologic interpretation between radiomic and pathomic features also should be central to future studies.

ACKNOWLEDGEMENTS This work was supported by "National Natural Science Foundation of China, grant number 81922040, 81930053, 81527805, 81772012", and "Beijing Natural Science Foundation, grant number 7182109", and "National Key R\&D Program of China, grant number 2017YFA0205200, 2017YFA0700401, 2016YFA0100902", and "Strategic Priority Research Program of Chinese Academy of Sciences, grant number XDB32030200, XDB01030200", and "Chinese Academy of Sciences, grant number QYZDJ-SSW-JSC005, KFJ-STS-ZDTP-059", and "Youth Innovation Promotion Association CAS, grant number 2019136".

DISCLOSURES All authors declare that they have no conflict of interest.

OPEN ACCESS This article is licensed under a Creative Commons Attribution 4.0 International License, which permits use, sharing, adaptation, distribution and reproduction in any medium or format, as long as you give appropriate credit to the original author(s) and the source, provide a link to the Creative Commons licence, and indicate if changes were made. The images or other third party material in this article are included in the article's Creative Commons licence, unless indicated otherwise in a credit line to the material. If material is not included in the article's Creative Commons licence and your intended use is not permitted by statutory regulation or exceeds the permitted use, you will need to obtain permission directly from the copyright holder. To view a copy of this licence, visit http://creativecommons. org/licenses/by/4.0/.

\section{REFERENCES}

1. Coit DG, Thompson JA, Algazi A, et al. Melanoma, version 2.2016, NCCN Clinical Practice Guidelines in Oncology. JNCCN J Natl Comp Cancer Network. 2016;4:450.

2. Renehan AG, Malcomson L, Emsley R, et al. Watch-and-wait approach versus surgical resection after chemoradiotherapy for patients with rectal cancer (the Oncore Project): a propensity-score matched cohort analysis. Lancet Oncol. 2016;2:174-83.

3. Trakarnsanga A, Gönen M, Shia J, et al. Comparison of tumor regression grade systems for locally advanced rectal cancer after multimodality treatment. J Natl Cancer Inst. 2014;106(10):dju248. https://doi.org/10.1093/jnci/dju248.

4. Cassidy J, Saltz L, Twelves C, et al. Efficacy of capecitabine versus 5-fluorouracil in colorectal and gastric cancers: a metaanalysis of individual data from 6171 patients. Ann Oncol. 2011;12:2604-9.

5. Liu ZY, Wang S, Dong D, et al. The applications of radiomics in precision diagnosis and treatment of oncology: opportunities and challenges. Theranostics. 2019;9(5):1303-22. https://doi.org/10.7 150/thno.30309.

6. Liu Z, Zhang X-Y, Shi Y-J, et al. Radiomics analysis for evaluation of pathological complete response to neoadjuvant chemoradiotherapy in locally advanced rectal cancer. Clin Cancer Res. 2017;23(23):7253-62.

7. Tang Z, Zhang X, Liu Z, et al. Quantitative analysis of diffusion weighted imaging to predict pathological good response to neoadjuvant chemoradiation for locally advanced rectal cancer. Radiother Oncol. 2019;132:100-8.

8. Zhou X, Yi Y, Liu Z, et al. Radiomics-based pretherapeutic prediction of non-response to neoadjuvant therapy in locally advanced rectal cancer. Ann SurgOncol. 2019;26(6):1676-84.

9. Shao L, Liu Z, Feng L, et al. Multiparametric MRI and whole slide image-based pretreatment prediction of pathological response to neoadjuvant chemoradiotherapy in rectal cancer: a multicenter radiopathomic study. Ann Surg Oncol. 2020. https://doi.org/10.12 45/s10434-020-08659-4.

Publisher's Note Springer Nature remains neutral with regard to jurisdictional claims in published maps and institutional affiliations. 